\title{
Convergence of greedy approximation I. General systems
}

\author{
by \\ S. V. Konyagin (Moscow) and V. N. Temlyakov (Columbia, SC)
}

\begin{abstract}
We consider convergence of thresholding type approximations with regard to general complete minimal systems $\left\{e_{n}\right\}$ in a quasi-Banach space $X$. Thresholding approximations are defined as follows. Let $\left\{e_{n}^{*}\right\} \subset X^{*}$ be the conjugate (dual) system to $\left\{e_{n}\right\}$; then define for $\varepsilon>0$ and $x \in X$ the thresholding approximations as $T_{\varepsilon}(x):=\sum_{j \in D_{\varepsilon}(x)} e_{j}^{*}(x) e_{j}$, where $D_{\varepsilon}(x):=\left\{j:\left|e_{j}^{*}(x)\right| \geq \varepsilon\right\}$. We study a generalized version of $T_{\varepsilon}$ that we call the weak thresholding approximation. We modify the $T_{\varepsilon}(x)$ in the following way. For $\varepsilon>0, t \in(0,1)$ we set $D_{t, \varepsilon}(x):=\left\{j: t \varepsilon \leq\left|e_{j}^{*}(x)\right|<\varepsilon\right\}$ and consider the weak thresholding approximations $T_{\varepsilon, D}(x):=T_{\varepsilon}(x)+\sum_{j \in D} e_{j}^{*}(x) e_{j}, D \subseteq D_{t, \varepsilon}(x)$. We say that the weak thresholding approximations converge to $x$ if $T_{\varepsilon, D(\varepsilon)}(x) \rightarrow x$ as $\varepsilon \rightarrow 0$ for any choice of $D(\varepsilon) \subseteq D_{t, \varepsilon}(x)$. We prove that the convergence set $W T\left\{e_{n}\right\}$ does not depend on the parameter $t \in(0,1)$ and that it is a linear set. We present some applications of general results on convergence of thresholding approximations to $A$-convergence of both number series and trigonometric series.
\end{abstract}

1. Introduction. Let $X$ be a quasi-Banach space (real or complex) with the quasi-norm $\|\cdot\|$ such that for all $x, y \in X$ we have $\|x+y\| \leq \alpha(\|x\|+\|y\|)$ and $\|t x\|=|t|\|x\|$. It is well known (see [KBR, Lemma 1.1]) that there is a $p, 0<p \leq 1$, such that

$$
\left\|\sum_{n} x_{n}\right\| \leq 4^{1 / p}\left(\sum_{n}\left\|x_{n}\right\|^{p}\right)^{1 / p}
$$

Let $\left\{e_{n}\right\} \subset X$ be a complete minimal system in $X$ with the conjugate (dual) system $\left\{e_{n}^{*}\right\} \subset X^{*}$. We assume that $\sup _{n}\left\|e_{n}^{*}\right\|<\infty$. This implies that for each $x \in X$ we have

$$
\lim _{n \rightarrow \infty} e_{n}^{*}(x)=0
$$

2000 Mathematics Subject Classification: 46A35, 46H05, 46G95.

This research was supported by the National Science Foundation Grant DMS 0200187 and by ONR Grant N00014-96-1-1003. 
Any element $x \in X$ has a formal expansion

$$
x \sim \sum_{n} e_{n}^{*}(x) e_{n},
$$

and various types of convergence of the series (1.3) can be studied. In this paper we deal with greedy type approximations with regard to the system $\left\{e_{n}\right\}$.

For any $x \in X$ we define the greedy ordering for $x$ as the map $\varrho: \mathbb{N} \rightarrow$ $\mathbb{N}$ such that $\left\{j: e_{j}^{*}(x) \neq 0\right\} \subset \varrho(\mathbb{N})$ and so that if $j<k$ then either $\left|e_{\varrho(j)}^{*}(x)\right|>\left|e_{\varrho(k)}^{*}(x)\right|$, or $\left|e_{\varrho(j)}^{*}(x)\right|=\left|e_{\varrho(k)}^{*}(x)\right|$ and $\varrho(j)<\varrho(k)$. The $m$ th greedy approximation is given by

$$
G_{m}(x):=G_{m}\left(x,\left\{e_{n}\right\}\right):=\sum_{j=1}^{m} e_{\varrho(j)}^{*}(x) e_{\varrho(j)} .
$$

The system $\left\{e_{n}\right\}$ is called a quasi-greedy system (see [KT1]) if there exists a constant $C$ such that $\left\|G_{m}(x)\right\| \leq C\|x\|$ for all $x \in X$ and $m \in \mathbb{N}$. Wojtaszczyk $[\mathrm{W}]$ proved that these are precisely the systems for which $\lim _{m \rightarrow \infty} G_{m}(x)=x$ for all $x$. If a quasi-greedy system $\left\{e_{n}\right\}$ is a basis then we say that $\left\{e_{n}\right\}$ is a quasi-greedy basis. It is clear that any unconditional basis is a quasi-greedy basis. We note that there are conditional quasi-greedy bases $\left\{e_{n}\right\}$ in some Banach spaces $[\mathrm{KT} 1, \mathrm{~W}]$. Hence, for such a basis $\left\{e_{n}\right\}$ there exists a permutation of $\left\{e_{n}\right\}$ which forms a quasi-greedy system but not a basis. This remark justifies the study of the class of quasi-greedy systems rather than the class of quasi-greedy bases.

Greedy approximations are close to thresholding approximations (sometimes they are called "thresholding greedy approximations"). Thresholding approximations are defined as

$$
T_{\varepsilon}(x)=\sum_{\left|e_{j}^{*}(x)\right| \geq \varepsilon} e_{j}^{*}(x) e_{j}, \quad \varepsilon>0 .
$$

Clearly, for any $\varepsilon>0$ there exists an $m$ such that $T_{\varepsilon}(x)=G_{m}(x)$. Therefore, if $\left\{e_{n}\right\}$ is a quasi-greedy system then

$$
\forall x \in X \quad \lim _{\varepsilon \rightarrow 0} T_{\varepsilon}(x)=x .
$$

Conversely, following Remark from [W, pp. 296-297], it is easy to show that the condition (1.4) implies that $\left\{e_{n}\right\}$ is a quasi-greedy system.

The following weak type greedy algorithm was considered in [T1]. Let $t \in(0,1]$ be a fixed parameter. For a given system $\left\{e_{n}\right\}$ and a given $x \in X$ denote by $\Lambda_{m}(t)$ any set of $m$ indices such that

$$
\min _{j \in \Lambda_{m}(t)}\left\|e_{j}^{*}(x) e_{j}\right\| \geq t \max _{j \notin \Lambda_{m}(t)}\left\|e_{j}^{*}(x) e_{j}\right\|
$$

and define 


$$
G_{m}^{t}(x):=G_{m}^{X, t}\left(x,\left\{e_{n}\right\}\right):=\sum_{j \in \Lambda_{m}(t)} e_{j}^{*}(x) e_{j} .
$$

We note that the greedy approximant $G_{m}^{t}(x)$ does not depend on normalization of the system $\left\{e_{n}\right\}$, and the previously defined greedy approximant $G_{m}(x)$ does depend on normalization. Usually we will denote by $\left\{e_{n}\right\}$ a general system and by $\left\{\psi_{n}\right\}$ a normalized one or a system which can be assumed normalized without loss of generality. By $\sigma_{m}\left(x,\left\{e_{n}\right\}\right)_{X}$ we denote the best $m$-term approximation in $X$ of $x$ with regard to the system $\left\{e_{n}\right\}$.

It was proved in [T1] that if $X=L_{p}, 1<p<\infty$, and $\left\{e_{n}\right\}$ is the Haar system $\mathcal{H}$, then for any $f \in L_{p}$,

$$
\left\|f-G_{m}^{L_{p}, t}(f, \mathcal{H})\right\|_{p} \leq C(p, t) \sigma_{m}(f, \mathcal{H})_{p} .
$$

This result motivated us to introduce a concept of greedy basis (see [KT1]).

Definition 1.1. We call a normalized basis $\Psi$ a greedy basis if for every $x \in X$ there exists a realization $\left\{G_{m}^{X, 1}(x, \Psi)\right\}$ such that

$$
\left\|x-G_{m}^{X, 1}(x, \Psi)\right\|_{X} \leq G \sigma_{m}(x, \Psi)_{X}
$$

with a constant independent of $x$ and $m$.

We note here that the proof of $[\mathrm{T} 1,(1.5)]$ works for any greedy basis in place of the Haar system $\mathcal{H}$. Thus for any greedy basis $\Psi$ of a Banach space $X$ and any $t \in(0,1]$ we have, for each $x \in X$,

$$
\left\|x-G_{m}^{X, t}(x, \Psi)\right\|_{X} \leq C(t) \sigma_{m}(x, \Psi)_{X} .
$$

This means that for greedy bases we have more flexibility in constructing nearly best $m$-term approximants. Similarly to the above, one can define weak thresholding approximations. Fix $t \in(0,1)$. For $\varepsilon>0$ define

$$
D_{t, \varepsilon}(x):=\left\{j: t \varepsilon \leq\left|e_{j}^{*}(x)\right|<\varepsilon\right\} .
$$

The weak thresholding approximations are defined as all possible sums

$$
T_{\varepsilon, D}(x)=\sum_{\left|e_{j}^{*}(x)\right| \geq \varepsilon} e_{j}^{*}(x) e_{j}+\sum_{j \in D} e_{j}^{*}(x) e_{j},
$$

where $D \subseteq D_{t, \varepsilon}(x)$. We say that the weak thresholding algorithm converges for $x \in X$ and write $x \in W T\left\{e_{n}\right\}(t)$ if for any $D(\varepsilon) \subseteq D_{t, \varepsilon}$,

$$
\lim _{\varepsilon \rightarrow 0} T_{\varepsilon, D(\varepsilon)}(x)=x \text {. }
$$

It is clear that the above relation is equivalent to

$$
\lim _{\varepsilon \rightarrow 0} \sup _{D \subseteq D_{t, \varepsilon}(x)}\left\|x-T_{\varepsilon, D}(x)\right\|=0 .
$$

We shall prove in Section 2 (see Theorem 2.1) that the set $W T\left\{e_{n}\right\}(t)$ does not depend on $t$. Therefore, we can drop $t$ from the notation: $W T\left\{e_{n}\right\}=$ $W T\left\{e_{n}\right\}(t)$. 
It turns out that the weak thresholding algorithm has more regularity than the thresholding algorithm: we will see that the set $W T\left\{e_{n}\right\}$ is linear while $W T\left\{e_{n}\right\}(1)$ can be nonlinear (see [KT2, Remark 2.4]). On the other hand, by "weakening" the thresholding algorithm (making convergence stronger) we do not narrow the convergence set too much. It is known that for many natural classes of subsets $Y$ of a Banach space $X$ the convergence of $T_{\varepsilon}(x)$ to $x$ for all $x \in Y$ is equivalent to $Y \subseteq W T\left\{e_{n}\right\}$. In particular, it can be derived from [W, Proposition 3] that the above two conditions are equivalent for $Y=X$.

2. General properties of the weak thresholding algorithm. We suppose that $X$ and $\left\{e_{n}\right\}$ satisfy the conditions stated at the beginning of the paper.

Theorem 2.1. Let $t, t^{\prime} \in(0,1), x \in X$. Then the following conditions are equivalent:

(1) $\lim _{\varepsilon \rightarrow 0} \sup _{D \subseteq D_{t, \varepsilon}(x)}\left\|T_{\varepsilon, D}(x)-x\right\|=0$;

(2) $\lim _{\varepsilon \rightarrow 0} T_{\varepsilon}(x)=x$ and

$$
\lim _{\varepsilon \rightarrow 0} \sup _{D \subseteq D_{t, \varepsilon}(x)}\left\|\sum_{j \in D} e_{j}^{*}(x) e_{j}\right\|=0
$$

(3) $\lim _{\varepsilon \rightarrow 0} T_{\varepsilon}(x)=x$ and

$$
\lim _{\varepsilon \rightarrow 0} \sup _{\left|a_{j}\right| \leq 1}\left\|\sum_{\left.j \in D_{t, \varepsilon}(x)\right)}\right\| a_{j \in D_{t, \varepsilon}(x)} e_{j}^{*}(x) e_{j} \|=0
$$

(4) $\lim _{\varepsilon \rightarrow 0} T_{\varepsilon}(x)=x$ and

$$
\lim _{\varepsilon \rightarrow 0} \sup _{\left|b_{j}\right|<\varepsilon}\left\|\sum_{\left(j:\left|e_{j}^{*}(x)\right| \geq \varepsilon\right)}\right\| b_{j:\left|e_{j}^{*}(x)\right| \geq \varepsilon} b_{j} e_{j} \|=0 ;
$$

(5) $\lim _{\varepsilon \rightarrow 0} \sup _{D \subseteq D_{t^{\prime}, \varepsilon}(x)}\left\|T_{\varepsilon, D}(x)-x\right\|=0$.

Proof. The equivalence of (1) and (2) follows easily from the definitions of $T_{\varepsilon}(x)$ and $T_{\varepsilon, D}(x)$.

The condition (2) follows from (3) since for any $D \subseteq D_{t, \varepsilon}(x)$ we can take $a_{j}=1$ for $j \in D$ and $a_{j}=0$ for $j \notin D$. To prove the implication $(2) \Rightarrow(3)$ we use the following lemma essentially proven in [W, Proposition 3]. We note that if $X$ is a Banach space $X$, this lemma is trivial.

Lemma 2.1. There is a constant $C=C(\alpha)$ such that for any $x_{1}, \ldots, x_{n}$ $\in X$ we have

$$
\max _{\left|a_{j}\right| \leq 1}\left\|\sum_{j=1}^{n} a_{j} x_{j}\right\| \leq C \max _{a_{j} \in\{0,1\}}\left\|\sum_{j=1}^{n} a_{j} x_{j}\right\| .
$$


Proof of Lemma 2.1. Define

$$
M=\max _{a_{j} \in\{0,1\}}\left\|\sum_{j=1}^{n} a_{j} x_{j}\right\| .
$$

Let us estimate the $\operatorname{sum} \sum_{j=1}^{n} a_{j} x_{j}$ for $a_{j} \in[0,1]$ first. Let $a_{j}=\sum_{s=1}^{\infty} a_{j, s} 2^{-s}$, where $a_{j, s} \in\{0,1\}$, be a digital expansion of $a_{j}$. Then, using (1.1), we obtain

$$
\begin{aligned}
\left\|\sum_{j=1}^{n} a_{j} x_{j}\right\|^{p} & =\left\|\sum_{s=1}^{\infty} 2^{-s} \sum_{j=1}^{n} a_{j, s} x_{j}\right\|^{p} \leq 4 \sum_{s=1}^{\infty} 2^{-s p}\left\|\sum_{j=1}^{n} a_{j, s} x_{j}\right\|^{p} \\
& \leq 4 \sum_{s=1}^{\infty} 2^{-s p} M^{p}=\left(C_{1} M\right)^{p} .
\end{aligned}
$$

Hence,

$$
\left\|\sum_{j=1}^{n} a_{j} x_{j}\right\| \leq C_{1} \max _{b_{j} \in\{0,1\}}\left\|\sum_{j=1}^{n} b_{j} x_{j}\right\| .
$$

The case of arbitrary coefficients $\left|a_{j}\right| \leq 1$ can be easily reduced to the case $a_{j} \in[0,1]$ by using a representation $a_{j}=a_{j}^{(1)}-a_{j}^{(2)}$ with $a_{j}^{(1)} \in[0,1], a_{j}^{(2)} \in$ $[0,1]$ for $X$ real and a similar representation $a_{j}=a_{j}^{(1)}-a_{j}^{(2)}+i a_{j}^{(3)}-i a_{j}^{(4)}$ for $X$ complex, and Lemma 2.1 follows.

Applying Lemma 2.1 for the set $\left\{x_{1}, \ldots, x_{n}\right\}=\left\{e_{j}^{*}(x) e_{j}: j \in D_{t, \varepsilon}(x)\right\}$, we get

$$
\sup _{\left|a_{j}\right| \leq 1}\left\|\sum_{\left.j \in D_{t, \varepsilon}(x)\right)} a_{j \in D_{t, \varepsilon}(x)} a_{j} e_{j}^{*}(x) e_{j}\right\| \leq C\left\|\sup _{D \subseteq D_{t, \varepsilon}(x)} \sum_{j \in D} e_{j}^{*}(x) e_{j}\right\|,
$$

and therefore (2) implies (3). Thus, we have proved that (2) and (3) are equivalent.

We will prove that (3) follows from (4) by proving that (4) implies (2). Indeed, for any $D \subseteq D_{t, \varepsilon}(x)$ we set $b_{j}=t e_{j}^{*}(x)$ for $j \in D$, and $b_{j}=0$ for $j \notin D$. Then $\left|b_{j}\right|<t \varepsilon$, and, by (4),

$$
\sup _{D \subseteq D_{t, \varepsilon}(x)}\left\|\sum_{j \in D} b_{j} e_{j}\right\| \rightarrow 0
$$

as $\varepsilon \rightarrow 0$, and (2) holds.

Let us show that (3) implies (4). Let $x \in X$. For $u>0$ define

$$
\Phi(u):=\sup _{\left|a_{j}\right| \leq 1}\left\|\sum_{\left.j \in D_{t, u}(x)\right)} a_{j \in D_{t, u}(x)} e_{j}^{*}(x) e_{j}\right\| .
$$

Then $\lim _{u \rightarrow 0} \Phi(u)=0$ by (3). Take $b_{j}\left(j:\left|e_{j}^{*}(x)\right| \geq \varepsilon\right)$ with $\left|b_{j}\right|<\varepsilon$, and 
estimate the sum

$$
S=\sum_{j:\left|e_{j}^{*}(x)\right| \geq \varepsilon} b_{j} e_{j}
$$

We have

$$
S=\sum_{s=1}^{\infty} S_{s}, \quad S_{s}=\sum_{j: t^{-(s-1)} \varepsilon \leq\left|e_{j}^{*}(x)\right|<t^{-s} \varepsilon} b_{j} e_{j} .
$$

By (2.4) with $u=t^{-s} \varepsilon$ we get

$$
\left\|S_{s}\right\|=\left\|\sum_{j: t^{-(s-1)}} \sum_{\varepsilon \leq\left|e_{j}^{*}(x)\right|<t^{-s} \varepsilon} b_{j} e_{j}\right\| \leq t^{s-1} \Phi\left(t^{-s} \varepsilon\right) .
$$

By (1.1) and (2.5),

$$
\|S\|^{p} \leq 4 \sum_{s=1}^{\infty} t^{p(s-1)} \Phi\left(t^{-s} \varepsilon\right)^{p} .
$$

It follows from the properties of the function $\Phi$ that the right-hand side of (2.6) tends to 0 as $\varepsilon \rightarrow 0$. Hence, (4) holds.

Finally, note that the condition (4) does not depend on the choice of $t \in(0,1)$. This shows that (1) is equivalent to (5) and completes the proof of the theorem. $(0,1)$.

So, the set $W T\left\{e_{n}\right\}$ defined in Section 1 is indeed independent of $t \in$

TheOrem 2.2. The set $W T\left\{e_{n}\right\}$ is linear.

Proof. It is enough to prove that $x+y \in W T\left\{e_{n}\right\}$ whenever $x, y \in$ $W T\left\{e_{n}\right\}$. By Theorem 2.1 it is sufficient to consider a particular parameter $t \in(0,1)$. Let us specify $t=1 / 2$ and prove that

$$
\lim _{\varepsilon \rightarrow 0} \sup _{D \subseteq D_{1 / 2, \varepsilon}(x+y)}\left\|T_{\varepsilon, D}(x+y)-(x+y)\right\|=0 .
$$

Take $\varepsilon>0$ and $D \subseteq D_{1 / 2, \varepsilon}(x+y)$. We will estimate $\left\|T_{\varepsilon, D}(x+y)-(x+y)\right\|$. Let

$$
D_{1}=D \cup\left\{j:\left|e_{j}^{*}(x+y)\right| \geq \varepsilon\right\}, \quad D_{2}=\mathbb{N} \backslash D_{1} .
$$

Notice that $j \in D_{1}$ implies $\left|e_{j}^{*}(x+y)\right| \geq \varepsilon / 2$ and therefore $\left|e_{j}^{*}(x)\right| \geq \varepsilon / 4$ or $\left|e_{j}^{*}(y)\right| \geq \varepsilon / 4$. We have

$$
T_{\varepsilon, D}(x+y)=\sum_{j \in D_{1}} e_{j}^{*}(x+y) e_{j} .
$$

Consider the sets

$$
\begin{aligned}
A & :=\left\{j:\left|e_{j}^{*}(x)\right| \geq \varepsilon / 4,\left|e_{j}^{*}(y)\right|<\varepsilon / 4\right\}, \\
A^{\prime} & :=\left\{j:\left|e_{j}^{*}(y)\right| \geq \varepsilon / 4,\left|e_{j}^{*}(x)\right|<\varepsilon / 4\right\},
\end{aligned}
$$




$$
B:=\left\{j:\left|e_{j}^{*}(x)\right| \geq \varepsilon / 4,\left|e_{j}^{*}(y)\right| \geq \varepsilon / 4\right\} .
$$

It is clear that $D_{1} \subseteq A \cup A^{\prime} \cup B$. It is also clear that

$$
A \cup A^{\prime} \cup B=D_{1} \cup E \cup F,
$$

where

$$
\begin{aligned}
& E:=\left\{j:\left|e_{j}^{*}(x)\right| \geq \varepsilon / 4, j \in D_{2}\right\}, \\
& F:=\left\{j:\left|e_{j}^{*}(y)\right| \geq \varepsilon / 4,\left|e_{j}^{*}(x)\right|<\varepsilon / 4, j \in D_{2}\right\} .
\end{aligned}
$$

Define

$$
\begin{aligned}
S_{1} & =\sum_{\left\{j:\left|e_{j}^{*}(x)\right| \geq \varepsilon / 4\right\}} e_{j}^{*}(x) e_{j}, & S_{4} & =\sum_{j \in A^{\prime}} e_{j}^{*}(x) e_{j}, \\
S_{2} & =\sum_{\left\{j:\left|e_{j}^{*}(y)\right| \geq \varepsilon / 4\right\}} e_{j}^{*}(y) e_{j}, & S_{5} & =\sum_{j \in E} e_{j}^{*}(x+y) e_{j}, \\
S_{3} & =\sum_{j \in A} e_{j}^{*}(y) e_{j}, & S_{6} & =\sum_{j \in F} e_{j}^{*}(x+y) e_{j} .
\end{aligned}
$$

Then

$$
S_{1}+S_{2}+S_{3}+S_{4}=\sum_{j \in A \cup A^{\prime} \cup B} e_{j}^{*}(x+y) e_{j} .
$$

Taking into account this fact, (2.8), and (2.9), we see that

$$
T_{\varepsilon, D}(x+y)-(x+y)=\left(S_{1}-x\right)+\left(S_{2}-y\right)+S_{3}+S_{4}-S_{5}-S_{6} .
$$

The terms $S_{1}-x$ and $S_{2}-y$ tend to 0 as $\varepsilon \rightarrow 0$ since $x, y \in W T\left\{e_{n}\right\}$. The sums $S_{j}, j=3,4,5,6$, tend to 0 by the condition (4) of Theorem 2.1. This proves Theorem 2.2.

REMARK 2.1. Using the same technique as in the proofs of Theorems 2.1 and 2.2 one can show that the linear set $W T\left\{e_{n}\right\}$ equipped with the quasi-norm

$$
\|x \mid\|=\sup _{\varepsilon} \sup _{D \subseteq D_{t, \varepsilon}(x)}\left\|T_{\varepsilon, D}(x)\right\|
$$

is a quasi-Banach space embedded in $X$. The system $\left\{e_{n}\right\}$ is a quasi-greedy system in the space $\left(W T\left\{e_{n}\right\},\||\cdot|\|\right)$.

We note that the space $\left(W T\left\{e_{n}\right\},\|\| \cdot \| \mid\right)$ need not be a Banach space even if $X$ is. Moreover, we will show in Section 3 (see Theorem 3.2) that the quasi-norm ||$\cdot|| \mid$ is not necessarily equivalent to any norm. Thus it would be unnatural to restrict ourselves to Banach spaces in studying quasi-greedy systems.

Let us now discuss the convergence of $G_{m}^{X, t}(x, \Psi)$ for quasi-greedy bases. 
THEOREM 2.3. Let $\Psi$ be a normalized quasi-greedy basis for a Banach space $X$. Then for any fixed $t \in(0,1]$ and each $x \in X$,

$$
G_{m}^{X, t}(x, \Psi) \rightarrow x \quad \text { as } m \rightarrow \infty .
$$

Proof. Let

$$
G_{m}^{X, t}(x, \Psi)=\sum_{j \in \Lambda_{m}(t)} c_{j}(x) \psi_{j}=S_{\Lambda_{m}(t)}(x, \Psi) .
$$

We set

$$
\begin{gathered}
\alpha:=\max _{j \notin \Lambda_{m}(t)}\left|c_{j}(x)\right| \\
\Lambda_{m}^{1}:=\left\{j:\left|c_{j}(x)\right|>\alpha\right\} \subseteq \Lambda_{m}(t), \quad \Lambda_{m}^{2}:=\left\{j:\left|c_{j}(x)\right| \geq t \alpha\right\} \supseteq \Lambda_{m}(t) .
\end{gathered}
$$

Then

$$
S_{\Lambda_{m}(t)}(x, \Psi)=S_{\Lambda_{m}^{1}}(x, \Psi)+S_{\Lambda_{m}(t) \backslash \Lambda_{m}^{1}}(x, \Psi) .
$$

The assumption that $\Psi$ is quasi-greedy implies that

$$
S_{\Lambda_{m}^{1}}(x, \Psi) \rightarrow x \quad \text { as } m \rightarrow \infty .
$$

We will prove that

$$
\left\|S_{\Lambda_{m}(t) \backslash \Lambda_{m}^{1}}(x, \Psi)\right\| \rightarrow 0 \quad \text { as } m \rightarrow \infty .
$$

We note that

$$
S_{\Lambda_{m}(t) \backslash \Lambda_{m}^{1}}(x, \Psi)=S_{\Lambda_{m}(t) \backslash \Lambda_{m}^{1}}\left(\sum_{j: t \alpha \leq\left|c_{j}(x)\right| \leq \alpha} c_{j}(x) \psi_{j}, \Psi\right) .
$$

We need a lemma on properties of quasi-greedy systems.

Lemma 2.2. Let $\Psi$ be a normalized quasi-greedy basis. Then for any two finite sets $A \subseteq B$ of indices, and coefficients $0<t \leq\left|a_{j}\right| \leq 1, j \in B$, we have

$$
\left\|\sum_{j \in A} a_{j} \psi_{j}\right\| \leq C(X, \Psi, t)\left\|\sum_{j \in B} a_{j} \psi_{j}\right\| .
$$

Proof. The proof is based on the following known lemma (see [DKKT]), essentially due to Wojtaszczyk [W].

It will be convenient to define the quasi-greedy constant $K$ to be the least constant such that

$$
\left\|G_{m}(x)\right\| \leq K\|x\| \quad \text { and } \quad\left\|x-G_{m}(x)\right\| \leq K\|x\|, \quad x \in X .
$$

Lemma 2.3. Suppose $\Psi$ is a normalized quasi-greedy basis with a quasigreedy constant $K$. Then for any real numbers $a_{j}$ and any finite set $P$ of indices we have

$$
\left(4 K^{2}\right)^{-1} \min _{j \in P}\left|a_{j}\right|\left\|\sum_{j \in P} \psi_{j}\right\| \leq\left\|\sum_{j \in P} a_{j} \psi_{j}\right\| \leq 2 K \max _{j \in P}\left|a_{j}\right|\left\|\sum_{j \in P} \psi_{j}\right\| .
$$


Using this lemma, we get

$$
\left\|\sum_{j \in A} a_{j} \psi_{j}\right\| \leq 2 K\left\|\sum_{j \in A} \psi_{j}\right\| \leq(2 K)^{2}\left\|\sum_{j \in B} \psi_{j}\right\| \leq(2 K)^{4} t^{-1}\left\|\sum_{j \in B} a_{j} \psi_{j}\right\| .
$$

This proves Lemma 2.2 .

We continue the proof of Theorem 2.3. Define

$$
x_{\alpha}:=\sum_{j: t \alpha \leq\left|c_{j}(x)\right| \leq \alpha} c_{j}(x) \psi_{j} .
$$

Then by Lemma 2.2, from (2.11) we get

$$
\left\|S_{\Lambda_{m}(t) \backslash \Lambda_{m}^{1}}(x, \Psi)\right\| \leq C\left\|x_{\alpha}\right\| .
$$

It remains to remark that $\alpha \rightarrow 0$ as $m \rightarrow \infty$, and $x_{\alpha} \rightarrow 0$ as $\alpha \rightarrow 0$.

We note that the $m$ th greedy approximant $G_{m}\left(x,\left\{e_{n}\right\}\right)$ changes if we renormalize the system $\left\{e_{n}\right\}$ (replacing it by $\left\{\lambda_{n} e_{n}\right\}$ ). This gives us more flexibility in adjusting a given system $\left\{e_{n}\right\}$ for greedy approximation. Let us make one simple observation in this direction.

Proposition 2.1. Let $\Psi=\left\{\psi_{n}\right\}_{n=1}^{\infty}$ be a normalized basis for a Banach space $X$. Then the system $\left\{e_{n}\right\}_{n=1}^{\infty}$, where $e_{n}:=2^{n} \psi_{n}, n=1,2, \ldots$, is a quasi-greedy system in $X$.

Proof. For a given $x \in X$ set $\delta_{N}(x):=\sup _{n \geq N}\left|\psi_{n}^{*}(x)\right|$. Then

$$
\delta_{N}(x) \rightarrow 0 \quad \text { as } N \rightarrow \infty \text {. }
$$

For $\varepsilon>0$ we denote by $N(\varepsilon):=N(x, \varepsilon)$ the smallest integer $N$ satisfying $\left|\psi_{n}^{*}(x)\right|<2^{n} \varepsilon$ for $n \geq N+1$. By (2.12) we get

$$
\lim _{\varepsilon \rightarrow 0} 2^{N(\varepsilon)} \varepsilon=0 \text {. }
$$

Let

$$
T_{\varepsilon}(x)=\sum_{n \in D_{\varepsilon}} e_{n}^{*}(x) e_{n}
$$

Then by the definition of $e_{n}$ and $N(\varepsilon)$ we obtain $D_{\varepsilon} \subseteq[1, N(\varepsilon)]$. Therefore, defining

$$
S_{N}(x, \Psi):=\sum_{n=1}^{N} \psi_{n}^{*}(x) \psi_{n}
$$

we get

$$
\begin{aligned}
\left\|S_{N(\varepsilon)}(x, \Psi)-T_{\varepsilon}(x)\right\| & =\left\|\sum_{n \leq N(\varepsilon):\left|e_{n}^{*}(x)\right|<\varepsilon} e_{n}^{*}(x) e_{n}\right\| \\
& =\left\|\sum_{n \leq N(\varepsilon):\left|\psi_{n}^{*}(x)\right|<2^{n} \varepsilon} \psi_{n}^{*}(x) \psi_{n}\right\| \leq 2^{N(\varepsilon)+1} \varepsilon \rightarrow 0
\end{aligned}
$$

as $\varepsilon \rightarrow 0$. This completes the proof of Proposition 2.1. 
We apply Proposition 2.1 to the trigonometric system $\left\{\psi_{n}\right\}_{n \geq 0}$, where $\psi_{0}=1, \psi_{2 n-1}:=e^{i n t}, \psi_{2 n}:=e^{-i n t}, n=1,2, \ldots$ It is known (see [T2]) that this system is not a quasi-greedy system for $L_{p}(\mathbb{T})$ for $p \neq 2$. Proposition 2.1 implies that $\left\{2^{|n|} e^{i n t}\right\}$ is a quasi-greedy system for $L_{p}(\mathbb{T}), 1<p<\infty$.

Let us discuss relations between the weak thresholding algorithm $T_{\varepsilon, D}(x)$ and the weak greedy algorithm $G_{m}^{t}(x)$. We define a modification of $G_{m}^{t}(x)$ that coincides with $G_{m}^{t}(x)$ for a normalized system $\left\{e_{n}\right\}$ and is close to $G_{m}(x)$ for a general system when $t=1$. For a given system $\left\{e_{n}\right\}, t \in(0,1]$, $x \in X$ and $m \in \mathbb{N}$, we denote by $W_{m}(t)$ any set of $m$ indices such that

$$
\min _{j \in W_{m}(t)}\left|e_{j}^{*}(x)\right| \geq t \max _{j \notin W_{m}(t)}\left|e_{j}^{*}(x)\right|,
$$

and define

$$
\widetilde{G}_{m}^{t}(x):=\widetilde{G}_{m}^{t}\left(x,\left\{e_{n}\right\}\right):=S_{W_{m}(t)}(x):=\sum_{j \in W_{m}(t)} e_{j}^{*}(x) e_{j} .
$$

It is clear that for any $t \in(0,1]$ and any $D \subseteq D_{t, \varepsilon}(x)$ there exist $m$ and $W_{m}(t)$ satisfying $(2.13)$ such that

$$
T_{\varepsilon, D}(x)=S_{W_{m}(t)}(x) .
$$

Thus the convergence $\widetilde{G}_{m}^{t}(x) \rightarrow x$ as $m \rightarrow \infty$ implies the convergence $T_{\varepsilon, D}(x) \rightarrow x$ as $\varepsilon \rightarrow 0$ for any $t \in(0,1]$. We will now prove that for $t \in(0,1)$ the converse is also true.

Proposition 2.2. Let $t \in(0,1)$ and $x \in X$. Then the following two conditions are equivalent:

$$
\begin{array}{r}
\lim _{\varepsilon \rightarrow 0} \sup _{D \subseteq D_{t, \varepsilon}(x)}\left\|T_{\varepsilon, D}(x)-x\right\|=0 \\
\lim _{m \rightarrow \infty}\left\|\widetilde{G}_{m}^{t}(x)-x\right\|=0
\end{array}
$$

for any realization $\widetilde{G}_{m}^{t}(x)$.

Proof. The implication $(2.15) \Rightarrow(2.14)$ is simple and follows from a remark preceding Proposition 2.2. We prove that $(2.14) \Rightarrow(2.15)$. Set

$$
\varepsilon_{m}:=\max _{j \notin W_{m}(t)}\left|e_{j}^{*}(x)\right| .
$$

Clearly $\varepsilon_{m} \rightarrow 0$ as $m \rightarrow \infty$. We have

$$
\widetilde{G}_{m}^{t}(x)=T_{2 \varepsilon_{m}}(x)+\sum_{j \in D_{m}} e_{j}^{*}(x) e_{j}
$$

with $D_{m}$ having the following property: for any $j \in D_{m}$,

$$
t \varepsilon_{m} \leq\left|e_{j}^{*}(x)\right|<2 \varepsilon_{m} .
$$

Thus by condition (5) from Theorem 2.1 for $t^{\prime}=t / 2$ we obtain (2.15).

Proposition 2.2 is now proved. 
Proposition 2.2 and Theorem 2.1 imply that the convergence set of the weak greedy algorithm $\widetilde{G}_{m}^{t}(\cdot)$ does not depend on $t \in(0,1)$ and coincides with $W T\left\{e_{n}\right\}$. By Theorem 2.2 this set is linear.

Let us make a comment on the case $t=1$, not covered by Proposition 2.2. It is clear that $T_{\varepsilon}(x)=G_{m}(x)$ with some $m$, and therefore $G_{m}(x) \rightarrow x$ as $m \rightarrow \infty$ implies $T_{\varepsilon}(x) \rightarrow x$ as $\varepsilon \rightarrow 0$. It is also not difficult to understand that in general $T_{\varepsilon}(x) \rightarrow x$ as $\varepsilon \rightarrow 0$ does not imply $G_{m}(x) \rightarrow x$ as $m \rightarrow \infty$. This can be seen, for instance, by considering the trigonometric system in $L_{p}$, $p \neq 2$, and using the Rudin-Shapiro polynomials (see [T2]). However, if for the trigonometric system we put the Fourier coefficients with equal absolute values in a natural order (say, lexicographic), then in the case $1<p<\infty$ by the Riesz theorem we obtain convergence of $G_{m}(f)$ from convergence of $T_{\varepsilon}(f)$. The results of $[\mathrm{KS}]$ show that the situation is different for $p=1$. In this case the natural order does not help to derive convergence of $G_{m}(f)$ from convergence of $T_{\varepsilon}(f)$.

3. A-convergence of number series. A series $\sum_{n} a_{n}, a_{n} \in \mathbb{C}$, is said to $A$-converge to $s \in \mathbb{C}$ if the following conditions hold:

$$
\begin{gathered}
\lim _{\varepsilon \rightarrow 0^{+}} \sum_{n:\left|a_{n}\right| \geq \varepsilon} a_{n}=s ; \\
\lim _{\varepsilon \rightarrow 0^{+}} \varepsilon\left|\left\{n:\left|a_{n}\right| \geq \varepsilon\right\}\right|=0 .
\end{gathered}
$$

We then write

$$
\text { (A) } \sum_{n} a_{n}=s .
$$

The notion of $A$-convergent series has been studied in [U2]; see also [U3]. It is similar to the well known notion of the $A$-integral (see, e.g., [U1]). We show that $A$-convergence can be treated as weak thresholding convergence of number series. Recall that $c_{0}$ is the space of sequences convergent to zero:

$$
c_{0}=\left\{x=\left(x^{0}, x^{1}, \ldots\right): x^{n} \in \mathbb{C}, \lim _{n \rightarrow \infty} x^{n}=0\right\},
$$

with the norm of $x \in c_{0}$ defined as $\|x\|=\max _{n}\left|x_{n}\right|$. It is known that

$$
c_{0}^{*}=l_{1}=\left\{\left(x^{0}, x^{1}, \ldots\right): x^{n} \in \mathbb{C},\|x\|=\sum_{n=0}^{\infty}\left|x^{n}\right|<\infty\right\} .
$$

Consider the system $\left\{e_{n}\right\}_{n \in \mathbb{N}} \subset c_{0}$ defined as $e_{n}^{0}=e_{n}^{n}=1, e_{n}^{j}=0$ for $j \neq 0, n$. It is clear that $\left\{e_{n}\right\}$ is a minimal system. It is also easy to see that $\left\{e_{n}\right\}$ is complete in $c_{0}$. For instance, for the coordinate vectors $u_{n}$ $\left(u_{n}^{n}=1, u_{n}^{j}=0\right.$ for $\left.j \neq n\right), n=0,1, \ldots$, we have

$$
\left\|u_{0}-\frac{1}{m} \sum_{n=1}^{m} e_{n}\right\|_{c_{0}} \leq \frac{1}{m}
$$




$$
u_{n}=e_{n}-u_{0}, \quad n=1,2, \ldots
$$

The elements $e_{n}^{*}$ of the conjugate system are $e_{n}^{*}=u_{n}, n=1,2, \ldots$ Thus, the formal expansion (1.2) takes the form

$$
x \sim \sum_{n=1}^{\infty} x^{n} e_{n}
$$

Clearly, this expansion converges to $x$ for $x \in c_{0}$ satisfying

$$
x^{0}=\sum_{n=1}^{\infty} x^{n} \text {. }
$$

THEOREM 3.1. Define the system $\left\{e_{n}\right\}_{n \in \mathbb{N}} \subset c_{0}$ as $e_{n}^{0}=e_{n}^{n}=1, e_{n}^{j}=0$ for $j \neq 0, n$. Let $\sum_{n \in \mathbb{N}} a_{n}$ be a number series with $\lim _{n \rightarrow \infty} a_{n}=0$, and let $s \in \mathbb{C}, t \in(0,1)$. Then the following conditions are equivalent:

(1) the series $\sum_{n} a_{n} A$-converges to $s$;

(2) $\lim _{\varepsilon \rightarrow 0} \sup _{D \subseteq D_{t, \varepsilon}}\left|T_{\varepsilon, D}-s\right|=0$, where

$$
D_{t, \varepsilon}=\left\{j: t \varepsilon \leq\left|a_{j}\right|<\varepsilon\right\}, \quad T_{\varepsilon, D}=\sum_{\left|a_{j}\right| \geq \varepsilon} a_{j}+\sum_{j \in D} a_{j} ;
$$

(3) the element $x \in c_{0}$ defined as $x=\left(s, a_{1}, a_{2}, \ldots\right)$ belongs to $W T\left\{e_{n}\right\}$.

Proof. We begin by proving that $(1) \Rightarrow(2)$. Using (3.2) we get, for any $D \subseteq D_{t, \varepsilon}$,

$$
\left|\sum_{j \in D} a_{j}\right| \leq \sum_{j \in D_{t, \varepsilon}}\left|a_{j}\right| \leq \sum_{j:\left|a_{j}\right| \geq t \varepsilon} \varepsilon=o(1 / \varepsilon) \varepsilon=o(1) .
$$

Therefore, taking into account (3.1) we get

$$
\sup _{D \subseteq D_{t, \varepsilon}}\left|T_{\varepsilon, D}-s\right|=o(1) .
$$

We now prove the implication $(2) \Rightarrow(1)$. It is a corollary of the following lemma.

LEMMA 3.1. The property (2) from Theorem 3.1 implies

$$
\left|D_{t, \varepsilon}\right|=o(1 / \varepsilon), \quad \varepsilon \rightarrow 0 .
$$

Proof of Lemma 3.1. Note that we can take $D^{\prime} \subseteq D_{t, \varepsilon}$ such that

$$
\left|\sum_{j \in D^{\prime}} a_{j}\right| \geq \frac{1}{4} \sum_{j \in D_{t, \varepsilon}}\left|a_{j}\right|
$$

Indeed, for $u \in \mathbb{R}$ set $u_{+}=\max (0, u)$. For any $z \in \mathbb{C}$ we have $|z| \leq$ $(\Re z)_{+}+(-\Re z)_{+}+(\Im z)_{+}+(-\Im z)_{+}$. Therefore, at least one of the following 
inequalities holds:

$$
\begin{aligned}
\sum_{j \in D_{t, \varepsilon}}\left(\Re a_{j}\right)_{+} & \geq \frac{1}{4} \sum_{j \in D_{t, \varepsilon}}\left|a_{j}\right|, \\
\sum_{j \in D_{t, \varepsilon}}\left(-\Re a_{j}\right)_{+} & \geq \frac{1}{4} \sum_{j \in D_{t, \varepsilon}}\left|a_{j}\right|, \\
\sum_{j \in D_{t, \varepsilon}}\left(\Im a_{j}\right)_{+} & \geq \frac{1}{4} \sum_{j \in D_{t, \varepsilon}}\left|a_{j}\right|, \\
\sum_{j \in D_{t, \varepsilon}}\left(-\Im a_{j}\right)_{+} & \geq \frac{1}{4} \sum_{j \in D_{t, \varepsilon}}\left|a_{j}\right| .
\end{aligned}
$$

If, say, (3.5) holds, then for $D^{\prime}=\left\{j \in D_{t, \varepsilon}: \Re a_{j} \geq 0\right\}$ we have

$$
\left|\sum_{j \in D^{\prime}} a_{j}\right| \geq \sum_{j \in D^{\prime}} \Re a_{j}=\sum_{j \in D_{t, \varepsilon}}\left(\Re a_{j}\right)_{+},
$$

and (3.4) holds. Other cases are studied similarly.

Thus, specifying $D=\emptyset$ and $D=D^{\prime}$ we deduce from (2) that

$$
\sum_{j \in D_{t, \varepsilon}}\left|a_{j}\right| \rightarrow 0 \quad(\varepsilon \rightarrow 0) \text {. }
$$

Using the fact that $\left|a_{j}\right| \geq t \varepsilon$ for $j \in D_{t, \varepsilon}$ we obtain

$$
\left|D_{t, \varepsilon}\right|=o(1 / \varepsilon) \quad(\varepsilon \rightarrow 0) .
$$

Similarly to the proof of the implication $(3) \Rightarrow(4)$ in Theorem 2.1 we hence obtain

$$
\left|\left\{j:\left|a_{j}\right| \geq \varepsilon\right\}\right|=o(1 / \varepsilon) .
$$

So, (3.2) is proved. The property (3.1) follows directly from (2) (take $D=\emptyset$ ).

We continue the proof of Theorem 3.1. The equivalence of the conditions (2) and (3) follows easily from the definition of the weak thresholding approximation. Theorem 3.1 is proved.

REMARK 3.1. In Theorem 3.1 we indexed (enumerated) the elements of the series $\sum_{n} a_{n}$ by the set of positive integers. Actually, this is not essential, we can assume that $n$ runs over any countable set.

The following corollary of Theorems 2.2 and 3.1 has been proved in [U2].

Corollary 3.1. The set of A-convergent series is linear. Moreover,

$$
\text { (A) } \sum_{n}\left(a_{n}+b_{n}\right)=(A) \sum_{n} a_{n}+(A) \sum_{n} b_{n} .
$$

REMARK 3.2. One can see from the proof of Theorem 3.1 that for any $t \in(0,1)$ the quasi-norm $\|\cdot \cdot\|_{t}$ in the space $Y=W T\left\{e_{n}\right\} \in c_{0}$ defined as 
in Remark 2.1,

$$
\||| x \mid\|_{t}=\sup _{\varepsilon} \sup _{D \subseteq D_{t, \varepsilon}(x)}\left\|T_{\varepsilon, D}(x)\right\|
$$

is equivalent to the quasi-norm

$$
\||| x||=\max \left(\left|x^{0}\right|, \sup _{\varepsilon} \varepsilon\left|\left\{n \geq 1:\left|x^{n}\right| \geq \varepsilon\right\}\right|\right) .
$$

Also, a quasi-norm in $Y$ can be treated as a quasi-norm in the space of $A$-convergent series.

Theorem 3.2. The quasi-norm $\|\mid \cdot\|$ in the space $Y=W T\left\{e_{n}\right\} \in c_{0}$ is not equivalent to any norm.

Proof. It is sufficient to show that for any $M>0$ there exist a positive integer $m$ and $x_{1}, \ldots, x_{m} \in Y$ such that

$$
\begin{gathered}
\|\| x_{j} \| \leq 1 \quad(j=1, \ldots, m), \\
\left\|\frac{1}{m} \sum_{j=1}^{m} x_{j}\right\|>M .
\end{gathered}
$$

Take an even $m \in \mathbb{N}$ and set $x_{j}^{n}=0$ for $n>m$, and $x_{j}^{n}=(-1)^{n} / k$ for $1 \leq n \leq m$, where $k \in\{1, \ldots, m\}$ is defined as $k \equiv n+j(\bmod m)$, $x_{j}^{0}=\sum_{n=1}^{m} x_{j}^{n}$. It is easy to see that all the elements $x_{j}=\left(x_{j}^{0}, x_{j}^{1}, \ldots\right)$ satisfy (3.10). Further, for $x=m^{-1} \sum_{j=1}^{m} x_{j}=\left(x^{0}, x^{1}, \ldots\right)$ we have

$$
\left|x^{n}\right|=\frac{1}{m} \sum_{k=1}^{m} \frac{1}{k} \quad(n=1, \ldots, m) .
$$

Therefore, $\left|\|x \mid\| \geq \sum_{k=1}^{m} 1 / k\right.$, and (3.11) holds for sufficiently large $m$. The proof of Theorem 3.2 is complete.

4. A-convergence of trigonometric series. In this section we use the results of the previous section to study the $A$-convergence of trigonometric series. The main results of this section concern the univariate case. However, we begin with the multivariate case. Consider a periodic function $f \in C\left(\mathbb{T}^{d}\right)$, defined on the $d$-dimensional torus $\mathbb{T}^{d}$. Denote the Fourier coefficients of $f$ by

$$
\widehat{f}(k):=(2 \pi)^{-d} \int_{\mathbb{T}^{d}} f(x) e^{-i(k, x)} d x .
$$

We will discuss the pointwise convergence of the Fourier expansion

$$
f(x) \sim \sum_{k \in \mathbb{Z}^{d}} \widehat{f}(k) e^{i(k, x)} .
$$


We can define weak thresholding approximations $T_{\varepsilon, D}(f)$ of the function $f$ with respect to the trigonometric system $\left\{e^{i(k, x)}\right\}$. Theorem 3.1 and Remark 3.1 give us the following criteria for pointwise $A$-convergence of (4.1).

Theorem 4.1. Let $f \in C\left(\mathbb{T}^{d}\right), x \in \mathbb{T}^{d}$, and $t \in(0,1)$. Then the following conditions are equivalent:

(1) the series $\sum_{k \in \mathbb{Z}^{d}} \widehat{f}(k) e^{i(k, x)}$ A-converges to $f(x)$;

$$
\lim _{\varepsilon \rightarrow 0} \sup _{D \subseteq D_{t, \varepsilon}(x)}\left|T_{\varepsilon, D}(f)(x)-f(x)\right|=0 .
$$

From now on we consider only the univariate case $d=1$. For a real function $f \in C(\mathbb{T})$ we can write its Fourier series in the real form:

$$
f \sim \sum_{n \in \mathbb{Z}_{+}} B_{n}(x),
$$

where $B_{0}=\widehat{f}(0), B_{n}(x)=\widehat{f}(n) e^{i n x}+\widehat{f}(-n) e^{-i n x}$ for $n>0$. The problem of pointwise $A$-convergence of Fourier series has been studied in [U2]. We will study relations between $A$-convergence of the complex expansion (4.1) and the real expansion (4.2) of Fourier series. In particular, we will prove that $A$-convergence of (4.1) implies $A$-convergence of (4.2). For $f \in C(\mathbb{T})$ we denote by $A_{\mathrm{c}}(f)\left(\operatorname{resp} . A_{\mathrm{r}}(f)\right)$ the set of points $x \in \mathbb{T}$ at which the series $\sum_{n \in \mathbb{Z}} \widehat{f}(n) e^{i n x}$ (resp. $\left.\sum_{n \in \mathbb{Z}_{+}} B_{n}(x)\right) A$-converges to $f(x)$.

Observe first that if $A_{\mathrm{c}}(f) \neq \emptyset$ then

$$
\lim _{\varepsilon \rightarrow 0^{+}} \varepsilon|\{k:|\widehat{f}(k)| \geq \varepsilon\}|=0 .
$$

Indeed, let $x \in A_{\mathrm{c}}(f)$. Then by (3.2) we get (4.3).

Theorem 4.2. Let $f \in C(\mathbb{T})$ be a real function. Then either $A_{\mathrm{c}}(f)=\emptyset$ or $A_{\mathrm{c}}(f)=A_{\mathrm{r}}(f)$. Moreover, if the measure of $A_{\mathrm{r}}(f)$ is positive then $A_{\mathrm{c}}(f)=$ $A_{\mathrm{r}}(f)$.

Proof. We first prove that if $A_{\mathrm{c}}(f) \neq \emptyset$ then $A_{\mathrm{c}}(f)=A_{\mathrm{r}}(f)$. Take $x \in$ $A_{\mathrm{c}}(f)$. The series $\sum_{n \in \mathbb{Z}} \widehat{f}(n) e^{i n x}$ and $\sum_{n \in \mathbb{Z}} \widehat{f}(-n) e^{-i n x} A$-converge to $f(x)$. By Corollary 3.1, their sum must be $A$-convergent to $2 f(x)$. This means that

$$
\text { (A) } \sum_{n \in \mathbb{Z}_{+}} 2 B_{n}(x)=2 f(x)
$$

or $x \in A_{\mathrm{r}}(f)$.

Conversely, take $x \in A_{\mathrm{r}}(f)$ and $\varepsilon>0$. We have

$$
\lim _{\varepsilon \rightarrow 0^{+}} \sum_{n \in \mathbb{Z}_{+}:\left|B_{n}(x)\right| \geq \varepsilon} B_{n}(x)=f(x) .
$$


Write

$$
\sum_{n \in \mathbb{Z}_{+}:\left|B_{n}(x)\right| \geq \varepsilon} B_{n}(x)-f(x)=S_{1}+S_{2}
$$

where

$$
\begin{aligned}
S_{1} & =\sum_{n \in \mathbb{Z}_{+}:\left|B_{n}(x)\right| \geq \varepsilon} B_{n}(x)-\sum_{n \in \mathbb{Z}:|\widehat{f}(n)| \geq \varepsilon / 2} \widehat{f}(n) e^{i n x}, \\
S_{2} & =\sum_{n \in \mathbb{Z}:|\widehat{f}(n)| \geq \varepsilon / 2} \widehat{f}(n) e^{i n x}-f(x) .
\end{aligned}
$$

We need to prove that

$$
S_{2}=o(1)
$$

For $S_{1}$ we have the following estimate:

$$
\left|S_{1}\right| \leq \sum_{\substack{n \in \mathbb{Z}_{+}:\left|B_{n}(x)\right|<\varepsilon \\|\widehat{f}(n)| \geq \varepsilon / 2}}\left|B_{n}(x)\right| \leq \sum_{n \in \mathbb{Z}_{+}:|\widehat{f}(n)| \geq \varepsilon / 2} \varepsilon=\varepsilon o(1 / \varepsilon)=o(1) .
$$

The relation (4.6) follows from (4.4), (4.5), (4.7). By (4.3) and (4.6), we have $x \in A_{\mathrm{c}}(f)$.

We proceed to the proof of the second part of Theorem 4.2. Taking into account the part already proved, we conclude that it is sufficient to prove that if $A_{\mathrm{c}}(f)=\emptyset$ then $\operatorname{mes}\left(A_{\mathrm{r}}(f)\right)=0$. We note that in the first part we have proved that if (4.3) is satisfied then $A_{\mathrm{c}}(f)=A_{\mathrm{r}}(f)$. Thus, it is sufficient to show that if (4.3) is not satisfied then $\operatorname{mes}\left(A_{\mathrm{r}}(f)\right)=0$. We will prove that if (4.3) is not satisfied then the relation

$$
\lim _{\varepsilon \rightarrow 0^{+}} \varepsilon\left|\left\{n:\left|B_{n}(x)\right| \geq \varepsilon\right\}\right|=0
$$

does not hold for almost all $x \in \mathbb{T}$. This follows from the assertion below, which is a generalization of the classical Denjoy-Lusin theorem [Z, p. 232].

THEOREM 4.3. Let $X$ be a quasi-Banach space of sequences $z:=\left\{z_{n}\right\}_{n=0}^{\infty}$ with the following properties:

(1) if $z \in X$ and $\left|y_{n}\right| \leq\left|z_{n}\right|$ for all $n$ then $y:=\left\{y_{n}\right\} \in X$ and $\|y\| \leq\|z\|$;

(2) if $z \in X$ and $z^{N} \in X$ is defined as: $z_{n}^{N}=z_{n}$ for $n \leq N, z_{n}^{N}=0$ for $n>N$, then

$$
\left\|z^{M}-z^{N}\right\| \rightarrow 0 \quad(M, N \rightarrow \infty) .
$$

Let $\sum_{n \in \mathbb{Z}} \widehat{f}(n) e^{i n x}$ be a trigonometric series, $|\widehat{f}(-n)|=|\widehat{f}(n)|, x \in \mathbb{T}$, $B_{0}=\widehat{f}(0), B_{n}(x)=\widehat{f}(n) e^{i n x}+\widehat{f}(-n) e^{-i n x}$ for $n>0$, and $E$ be a subset of $\mathbb{T}$ of positive measure. If $\left\{B_{n}(x)\right\} \in X$ for all $x \in E$, then $\left\{f_{n}\right\}_{n=0}^{\infty}:=$ $\{\widehat{f}(n)\}_{n=0}^{\infty} \in X$. 
In the case $X=l_{1}$ Theorem 4.3 is the Denjoy-Lusin theorem. Applying Theorem 4.3 to the space of sequences $\left\{a_{n}\right\}$ satisfying (3.2) with the quasinorm $\sup _{\varepsilon>0} \varepsilon\left|\left\{n:\left|a_{n}\right| \geq \varepsilon\right\}\right|$, we complete the proof of Theorem 4.2.

Proof of Theorem 4.3. By (2), for any $x \in E$,

$$
\lim _{M, N \rightarrow \infty}\left\|\left\{B_{n}^{M}(x)\right\}-\left\{B_{n}^{N}(x)\right\}\right\|=0 .
$$

Note that the mappings $x \mapsto\left\{\left|B_{n}^{M}(x)\right|\right\}$ and $x \mapsto\left\{\left|B_{n}^{N}(x)\right|\right\}$ are continuous. By (1) the mappings $x \mapsto\left\|\left\{\left|B_{n}^{M}(x)\right|\right\}\right\|$ and $x \mapsto\left\|\left\{\left|B_{n}^{N}(x)\right|\right\}\right\|$ are also continuous. For $x \in E$ define

$$
g_{N}(x):=\sup _{M>N}\left\|\left\{B_{n}^{M}(x)\right\}-\left\{B_{n}^{N}(x)\right\}\right\| .
$$

These are measurable functions such that for each $x \in E$ (see (4.8)),

$$
\lim _{N \rightarrow \infty} g_{N}(x)=0 .
$$

By Egorov's theorem we can take a subset $E_{1} \subset E$ of positive measure such that the convergence in (4.8) is uniform. Thus,

$$
\lim _{M, N \rightarrow \infty} \sup _{x \in E_{1}}\left\|\left\{B_{n}^{M}(x)\right\}-\left\{B_{n}^{N}(x)\right\}\right\|=0 .
$$

Consider $n$ with $|\widehat{f}(n)|>0$. There exists $x_{0} \in \mathbb{T}$ such that $e^{2 \pi i n x_{0}}=$ $-\widehat{f}(-n) / \widehat{f}(n)$, or $B_{n}\left(x_{0}\right)=0$. For $x \in \mathbb{T}$ we have

$$
\left|B_{n}(x)\right|=2\left|\sin \left(n\left(x-x_{0}\right)\right)\right||\widehat{f}(n)| .
$$

This implies

$$
\operatorname{mes}\left\{x \in \mathbb{T}:\left|B_{n}(x)\right| /|\widehat{f}(n)| \leq 2 \sin u\right\}=4 u \quad(0 \leq u \leq \pi / 2) .
$$

Therefore,

$$
\int_{E_{1}}\left|B_{n}(x)\right| \geq C|\widehat{f}(n)|, \quad C=\int_{0}^{\text {mes }_{1}} 2 \sin (u / 4) d u .
$$

For arbitrary positive integers $M$ and $N$ with $M>N$ we find from (4.10) and the condition (1) of the theorem that

$$
\left\|\int_{E_{1}}\left(\left\{\left|B_{n}^{M}(x)\right|\right\}-\left\{\left|B_{n}^{N}(x)\right|\right\}\right) d x\right\| \geq C\left\|\left\{\left|f_{n}^{M}\right|\right\}-\left\{\left|f_{n}^{N}\right|\right\}\right\| .
$$

It follows from (1.1) that

$$
\left\|\int_{E_{1}}\left(\left\{\left|B_{n}^{M}(x)\right|\right\}-\left\{\left|B_{n}^{N}(x)\right|\right\}\right) d x\right\|^{p} \leq 4 \int_{E_{1}}\left\|\left\{\left|B_{n}^{M}(x)\right|\right\}-\left\{\left|B_{n}^{N}(x)\right|\right\}\right\|^{p} d x .
$$

Combining this inequality with (4.11) we obtain

$$
\left\|\left\{\left|f_{n}^{M}\right|\right\}-\left\{\left|f_{n}^{N}\right|\right\}\right\|^{p} \leq 4 C^{-p} \int_{E_{1}}\left\|\left\{\left|B_{n}^{M}(x)\right|\right\}-\left\{\left|B_{n}^{N}(x)\right|\right\}\right\|^{p} d x,
$$


and by (4.9),

$$
\lim _{M, N \rightarrow \infty}\left\|\left\{\left|f_{n}^{M}\right|\right\}-\left\{\left|f_{n}^{N}\right|\right\}\right\|=0 .
$$

So, the sequence $\left\{\left|\widehat{f}(n)^{N}\right|\right\}$ is a Cauchy sequence. It has a limit $w \in X$. Consider the linear functional $e_{n}^{*}$ on $X$ defined by $e_{n}^{*}(y)=y_{n}$ for $y \in X$. We have

$$
w_{n}=e_{n}^{*}(w)=\lim _{N \rightarrow \infty} e_{n}^{*}\left(f_{n}^{N}\right)=f_{n} .
$$

Therefore, $\left\{f_{n}\right\}_{n=0}^{\infty}=w \in X$. This completes the proof of Theorem 4.3.

\section{References}

[DKKT] S. J. Dilworth, N. J. Kalton, D. Kutzarova, and V. N. Temlyakov, The thresholding greedy algorithm, greedy bases, and duality, IMI Preprint Series 23 (2001), 1-23.

[KBR] N. J. Kalton, N. T. Beck, and J. W. Roberts, An F-space Sampler, London Math. Soc. Lecture Note 5, Cambridge Univ. Press, Cambridge, 1984.

[KS] S. V. Konyagin and M. A. Skopina, Comparison of the $L_{1}$-norms of total and truncated exponential sums, Mat. Zametki 69 (2001), 699-707 (in Russian).

[KT1] S. V. Konyagin and V. N. Temlyakov, A remark on greedy approximations in Banach spaces, East J. Approx. 5 (1999), 1-15.

[KT2] - - C, Convergence of greedy approximation II. The trigonometric system, this volume, 161-184.

[T1] V. N. Temlyakov, The best $m$-term approximation and greedy algorithms, Adv. Comput. Math. 8 (1998), 249-265.

[T2] -, Greedy algorithm and m-term trigonometric approximation, Constr. Approx. 107 (1998), 569-587.

[U1] P. L. Ul'yanov, Integrals of Cauchy type, Trudy Mat. Inst. Steklova 60 (1961), 262-281 (in Russian).

[U2] - On the A-convergence of series, Dokl. Math. 60 (1999), 176-179.

[U3] -, On the properties of series, Dokl. Math. 62 (2000), 56-59 .

[W] P. Wojtaszczyk, Greedy algorithm for general biorthogonal systems, J. Approx. Theory 107 (2000), 293-314.

[Z] A. Zygmund, Trigonometric Series, Vol. 1, Cambridge Univ. Press, Cambridge, 1977.

Department of Mechanics and Mathematics

Moscow State University

119992 Moscow, Russia

E-mail: konyagin@ok.ru
Department of Mathematics University of South Carolina Columbia, SC 29208, U.S.A. E-mail: temlyak@math.sc.edu

Received October 3, 2002

Revised version May 5, 2003 